\title{
Uncertainty of the Shapley Value
}

\author{
Vladislav Kargin*†
}

November 3, 2018

\begin{abstract}
This paper introduces a measure of uncertainty in the determination of the Shapley value, illustrates it with examples, and studies some of its properties. The introduced measure of uncertainty quantifies random variations in a player's marginal contribution during the bargaining process. The measure is symmetric with respect to exchangeable substitutions in the players, equal to zero for dummy player, and convex in the game argument. The measure is illustrated by several examples of abstract games and an example from epidemiology.
\end{abstract}

${ }^{*}$ Cornerstone Research, 599 Lexington Avenue, New York, NY 10022, USA; slava@bu.edu

${ }^{\dagger}$ The views expressed within this article are the views of the author and not the views of Cornerstone Research. 
The dread of beatings! Dread of being late!

And, greatest dread of all, the dread of games!

Sir John Betjeman, "Summoned by Bells", ch.7

\section{Introduction}

An important problem in epidemiology is to assign weights to the factors that contribute to an increase in the incidence rate of a disease. For example, a higher incidence rate of a heart disease may be attributable to smoking, bad eating habits, or lack of physical exercise, and epidemiologists are interested in quantifying the relative degree of importance of these factors. There are different methods to perform the attribution, all of which must deal with the fact that the contribution of a factor to the incidence rate depends on the presence of the other factors. For instance, smoking may hypothetically have a relatively small impact on health unless it is accompanied by other factors. This difficulty is similar to the problems that arise in cooperative game theory, and in a recent paper Land and Gefeller have proposed an attribution according to the Shapley value, the standard solution concept from game theory. Specifically, they have studied the influence of smoking and three types of cholesterol level, LDL, HDL, and VLDL, on a certain heart disease, myocardial infarction. When all four factors are absent, the incidence rate of the disease is 77 percent lower than the incidence rate in the total population. The Shapley attribution for this

reduction is 21 percent for smoking, and 41,8 , and 8 percent for the cholesterol 
levels respectively. A natural question arises about the statistical significance of these attributions. The present paper proposes a measure of the uncertainty of the Shapley value based on the probabilistic interpretation of this game-theoretic concept.

The uncertainty of the Shapley value is going to be useful not only in practical applications similar to the epidemiological example above but also in theoretical problems. For example, following a line of research initiated by Shapley, Aumann (1975) has shown that as the number of participants in a market economy grows, the Shapley value of a game associated with this economy converges to the competitive equilibrium allocation. This convergence puts the concept of the competitive equilibrium on firm game-theoretical grounds, but only if it is presumed that the Shapley value reflects the results of the bargaining in the economy accurately. If the Shapley value provides the outcome of the bargaining with only a limited degree of certainty, then the natural question arises: What if the uncertainty of the Shapley value increases with the increase in the number of participants? Wouldn't it imply that the outcome of bargaining does not necessarily converge to competitive equilibrium? And if the uncertainty does decrease, then how does the rate of the decrease depend on the details of the market organization? Answering these questions is important for a better understanding of how the competitive equilibrium is achieved in market economies.

From another perspective, the Shapley value is used not only as a device to compute a fair allocation of resources among players but also as a tool to 
evaluate the prospect of playing a game. The bargaining uncertainty would provide an additional dimension for evaluating this prospect. Indeed, both experimental evidence and personal introspection suggest that participation in a bargaining situation implies undergoing the vagaries of the bargaining process, an experience that may be a source of pleasure or displeasure depending on the personality of the participant. A measure of bargaining uncertainty would serve as a useful tool to investigate this aspect of a game. Characterizing a game by both its value and uncertainty is similar to characterizing a weapon by its power and precision, or a financial stock by its expected return and risk. In all these situations the complexity of a real-world object cannot be compressed into just one dimension, it needs at least two for proper description.

So, how should the game uncertainty be measured? To answer this question, let us first examine the different frameworks that are used to define the Shapley value.

The shortest way to define the Shapley value is axiomatic. However, this approach is not suitable for measuring the Shapley value uncertainty. This is because the Shapley value is the unique solution satisfying all the axioms, and relaxation of any of them leads to an infinity of new solutions. In contrast, the probabilistic interpretation of the Shapley value suits the problem of defining uncertainty very well: the Shapley value is defined as the expectation of the marginal contribution to a random coalition, and it is straightforward to define the uncertainty as the variance of the marginal contribution. This is the way of defining uncertainty that the present paper propounds. 
The probabilistic interpretation of the Shapley value was sketched by Shapley in his seminal paper $((\underline{1953}))$. Later it was set on firm ground by Gul (1989), Evans (1996), and Hart and Mas-Colell (1996) in their work on non-cooperative foundations of the Shapley value, work that was inspired by Rubinstein's paper on the bargaining problem $(\underline{(1982)})$. Essentially, in all these probabilistic interpretations, the randomness of the model is the randomness of bargaining order and coalition formation.

To understand how the randomness in the order of bargaining introduces uncertainty about the outcome, it is useful to recall Rubinstein's model of the bargaining game. In this game two players aim to divide a pie that has unit value. The players are impatient and make offers in turn until some of them agrees to the other player's offer. The player who makes the first offer is determined by chance. Rubinstein has shown that there is a unique subgame-perfect equilibrium in this game. The ex-ante expected payoff of the game is one half, but the first player has an advantage and gets greater part of the pie. The uncertainty here arises because players initially do not know who makes the first offer, and a natural measure of uncertainty is the expectation of the squared difference between actual and expected payoffs.

The present paper generalizes this measure and illustrates with examples that the uncertainty of the Shapley value is an interesting object that behaves differently in different types of games. Some of its properties are parallel to the properties of the Shapley value. The linearity property is, however, replaced by convexity. It is also conjectured and proved for certain games that the 
uncertainty of the sum of two games is larger than the sum of the corresponding uncertainties.

The concept of uncertainty of a game outcome is not entirely new. Roth (1988) has pointed out that the Shapley value is insufficient for decision making purposes unless the players are risk-neutral to a specific kind of uncertainty in the strategic interactions, which he calls "strategic risk". He distinguishes this kind of risk from "ordinary risk" that typically arise in lotteries, and he characterizes the class of utility functions that may depend on this risk. Roth, however, does not attempt to introduce a quantitative measure of strategic risk, and that is the main contribution of the present paper.

The introduced measure provides a yardstick for both measuring the reliability of the Shapley value and quantifying the strategic risk. The main advantage of this measure is its simplicity. Future work will perhaps provide alternative measures of uncertainty based on more accurate analysis of bargaining randomness. The purpose of this paper is simply to bring attention to the importance of measuring this randomness and to suggest a possible solution.

The rest of the paper is organized as follows. Section 2 provides notation and basic definitions. Section 3 lists examples. Section 4 shows certain properties of the uncertainty concept, and Section 5 concludes.

\footnotetext{
${ }^{1}$ See also Roth (1977a) and Roth (1977b)
} 


\section{Notation and Definitions}

Let $A$ be a set of players: $A=\{1,2, \ldots N\}$. A game $G$ is a map from a set of all subsets of $A$ to real numbers: $2^{A} \rightarrow \mathbb{R}$. The interpretation of $G(S)$ is the payoff that the coalition of players in subset $S$ can achieve on their own.

Let $(X, \Omega, \mu)$ be a probability space and $f_{i}: X \rightarrow 2^{A-\{i\}}$ be a random variable that takes its values in the set of all subsets of $A-\{i\}$, and has the following distribution function:

$$
\mu\left\{f_{i}(X)=S\right\}=\frac{|S| !(N-|S|-1) !}{N !}
$$

Random variable $f_{i}$ can be interpreted as the random choice of coalition that player $i$ joins. The particular choice of its probability distribution is motivated by both the axiomatic approach and non-cooperative bargaining games that support the Shapley value.

Define the marginal contribution of player $i$ as a function $d_{i} G$ that acts on coalitions from $A-\{i\}$ in the following way:

$$
d_{i} G(S)=G(S+i)-G(S)
$$

Then the Shapley value of game $G$ for player $i$ is the expectation of his marginal contribution when the coalition is chosen randomly.

$$
V_{i}(G)=\mathbf{E}\left\{d_{i} G \circ f_{i}\right\}
$$

The Shapley uncertainty of game $G$ for player $i$ is defined as the variance of his marginal contribution:

$$
R_{i}(G)=\operatorname{Var}\left\{d_{i} G \circ f_{i}\right\}
$$


This definition easily generalizes to other, non-Shapley, values. Indeed, almost all reasonable values are probabilistic values (Weber (1988), which can be represented as the expectation of the player's marginal contribution with respect to a certain probability distribution imposed on coalitions. Of course, for different bargaining mechanisms the probability distributions may be different. For example, another popular value, the Banzhaf value, corresponds to the following probability distribution:

$$
\mu\left\{f_{i}(X)=S\right\}=\frac{1}{2^{N-1}} .
$$

For probabilistic values, it is natural to define the corresponding uncertainty as the variance of the marginal contribution. Most of the properties that will be derived below for the uncertainty of the Shapley value will also hold for the uncertainty of other probabilistic values. This paper, however, sticks with the Shapley value as the most popular in practical applications.

\section{$3 \quad$ Interpretation and Examples}

In many non-cooperative interpretations of the Shapley value, the actual payoff of a player is a weighted average of his marginal contribution to a random coalition and his expected payoff:

$$
\pi_{i}=\alpha\left(d_{i} G(S)\right)+(1-\alpha) V_{i}(G) .
$$

For instance, in the Rubinstein-Gul-type bargaining models the parameter $\alpha$ is related to player impatience. Then, the variance of player payoff is proportional 
to the Shapley uncertainty:

$$
\operatorname{Var}\left\{\pi_{i}\right\}=\alpha^{2} R_{i}(G)
$$

According to the Chebyshev inequality, it follows that

$$
\operatorname{Pr}\left\{\left|\frac{\pi_{i}}{V_{i}(G)}-1\right| \geqslant c\right\} \leqslant \alpha^{2} \frac{R_{i}(G)}{\left[V_{i}(G)\right]^{2}}
$$

that is, that the Shapley uncertainty provides a bound on the deviation of the actual payoff from its expected value. Moreover, if the marginal contributions are distributed approximately normally, which is likely to happen in large games, then the statistical results for normal distributions are applicable and the following inequality holds:

$$
\operatorname{Pr}\left\{\left|\pi_{i}-V_{i}(G)\right| \geqslant 1.96 \alpha \sqrt{R_{i}(G)}\right\} \leqslant 0.05
$$

So, the Shapley uncertainty can be interpreted as measuring the variability of the actual payoff.

Let me now present several examples.

\section{Example 3.1 Additive game}

In the additive game the value of a coalition is simply the sum of the values of individual players. The Shapley value for a player coincides with his individual value and, consequently, the risk is zero: Each player can guarantee his own value.

Example 3.2 Uncertainty may be different for different players. 
Let a three-player game be defined as follows. $G(\{1\})=0, G(\{2\})=3$, $G(\{3\})=6, G(\{1,2\})=24, G(\{2,3\})=18, G(\{1,3\})=21, G(\{1,2,3\})=60$.

Then,

$$
V_{1}=V_{2}=V_{3}=20,
$$

and

$$
R_{1}=299 ; R_{2}=230 ; R_{3}=155 .
$$

This example illustrates that players can have the same Shapley values but different Shapley uncertainties.

\section{Example 3.3 Majority game}

In the majority game the value of a coalition is $N$ if the size of the coalition is greater than half of the number of players, and 0 otherwise. For simplicity, let the number of players $N$ be odd. Then the Shapley value of the game for player $i$ is

$$
V_{i}(G)=1
$$

and the Shapley uncertainty is

$$
R_{i}(G)=N
$$

So the uncertainty of the majority game increases with an increase in the number of players. Intuitively this is because the marginal contribution of a player is non-zero only if his vote is pivotal in a random ordering of players, and in the majority game with a large number of players this situation is very rare. 
Example 3.4 Large production economy ${ }^{2}$

An economy consists of $N$ agents, of which $k N$ are capitalists and $(1-k) N$ are workers. Each capitalist owns a factory and each worker owns one unit of labor power. $K$ capitalists and $L$ workers can produce $\sqrt{K L}$ units of output. What is the value and the uncertainty of the game for the participants?

It is easy to check that for a random large coalition of size $X$, the frequency of capitalists is distributed according to the Gaussian law:

$$
\widehat{k} \sim \mathcal{N}\left(k, \frac{k}{X}\right)
$$

where $\widehat{k}$ is the sample frequency of capitalists, and $\mathcal{N}(E, V)$ is the notation for the Gaussian distribution with expectation $E$ and variance $V$. The marginal contribution of a worker added to this coalition is

$$
d_{w} G(S)=\frac{1}{2} \sqrt{\frac{\widehat{k}}{1-\widehat{k}}} .
$$

For a large $X$ we can compute expectation and variance of this expression, and averaging them over the coalition size, $X$, gives the value and the uncertainty of the game for a worker:

$$
\begin{aligned}
V_{w}(G) & =\frac{1}{2} \sqrt{\frac{k}{1-k}}, \\
R_{w}(G) & \sim \frac{1}{16(1-k)^{3}} \frac{\ln N}{N}(\text { where } N \rightarrow \infty) .
\end{aligned}
$$

\footnotetext{
${ }^{2}$ This example is based on an example of a production economy in Shapley and Shubik (1967) and Osborne and Rubinstein (1994)
} 
Similarly, for a capitalist we have

$$
\begin{aligned}
V_{c}(G) & =\frac{1}{2} \sqrt{\frac{1-k}{k}} \\
R_{c}(G) & \sim \frac{1}{16 k^{3}} \frac{\ln N}{N}(\text { where } N \rightarrow \infty) .
\end{aligned}
$$

In this simple example of production economy, the uncertainty of the game decreases as the size of the game grows. The intuition behind this is simple: As the number of participants grows, almost all coalitions have approximately the same structure as the total population. For these typical coalitions an agent has approximately the same marginal contribution, so his uncertainty is low.

\section{Example 3.5 Large Market Economy}

Suppose there are just two commodities in the economy, apples and bread. Assume there are $k N$ apple traders and $(1-k) N$ bread traders. Suppose also that initially each trader has one unit of commodity of his own type, and that the utility function of every trader is the same:

$$
u(a, b)=\sqrt{a b},
$$

where $a$ is the number of apples and $b$ is the quantity of bread. Assume that the utility is transferable.

Then a coalition of $A$ apple and $B$ bread traders has worth

$$
G(S)=\sqrt{A B}
$$

and the problem is completely analogous to the problem of Example 3.4 Thus, 
for the apple trader the value and the uncertainty are

$$
\begin{aligned}
V_{a}(G) & =\frac{1}{2} \sqrt{\frac{1-k}{k}} \\
R_{a}(G) & \sim \frac{1}{16 k^{3}} \frac{\ln N}{N}(\text { where } N \rightarrow \infty) .
\end{aligned}
$$

The expressions for the value and uncertainty of the bread trader are similar. Therefore, this example suggests that in the market economy, as well as in the production economy, the uncertainty goes to zero when the size of the economy increases.

\section{Example 3.6 Risk Factor Attribution}

The Shapley values for the epidemiological example from Land and Gefeller (1997) are $0.405,0.08,0074$ respectively for abnormal LDL, VLDL, and HDL cholesterol levels and 0.211 for smoking. The square roots of the corresponding Shapley uncertainties can be computed as $0.118,0.056,0.057,0.11$. The standard statistical test of significance shows that only the effects of abnormal LDL and smoking are significant at a 5 percent significance level.

\section{Properties of the Shapley Uncertainty}

Several of the properties of the Shapley uncertainty are similar to the properties of the Shapley value and follow directly from the definition. They are scaling, "zero risk for dummy", and symmetry properties:

Proposition 4.1 (Scaling) $R_{i}(t G)=t^{2} R_{i}(G)$ 
Proposition 4.2 (Dummy) If $G(X+i)=G(X)+G(i)$ for any $X$ that does not contain $i$ then $R_{i}(G)=0$.

Proposition 4.3 (Symmetry) If $G(X)=G(X-i+j)$ for any $X$ that contains $i$, then $R_{i}(G)=R_{j}(G)$.

For two-player games a stronger symmetry property holds. It states that in two-player games both players are always equally exposed to the Shapley uncertainty even if their values are different.

Proposition 4.4 (Strong Symmetry) For any 2-player game $R_{i}(G)=R_{j}(G)$.

For games with more than two players this property is not true but still the risks of different players cannot be too different, as the following proposition shows.

Proposition 4.5 For every $N$-player game $G$

$$
R_{i}(G) \leq(N-1) \sum_{j \neq i} R_{j}(G) .
$$

Proof: First, the Shapley uncertainty can be defined in an equivalent way. Let $\mathfrak{B}$ be the space of all orderings of elements in $A$. Define a map $p_{i}: \mathfrak{B} \rightarrow 2^{A}$ that takes an ordering to the subset of elements of $A$ that precedes element $i$ in the ordering, and let $\widetilde{d_{i} G}=d_{i} G \circ p_{i}$. Take a random variable $g: X \rightarrow \mathfrak{B}$ that has a uniform distribution on orderings. Then it is easy to check that:

$$
\begin{aligned}
f_{i} & =p_{i} \circ g, \\
V_{i}(G) & =\mathrm{E}\left(\widetilde{d_{i} G} \circ g\right), \\
R_{i}(G) & =\operatorname{Var}\left(\widetilde{d_{i} G} \circ g\right) .
\end{aligned}
$$


Second, for any ordering $b \in \mathfrak{B}$, the sum of marginal contributions of all players is equal to the value of the game, which is not random:

$$
\sum_{i} \widetilde{d_{i} G}(b)=G(A)
$$

It follows

$$
\operatorname{Var}\left(\widetilde{d_{i} G} \circ g\right)=\operatorname{Var}\left(\sum_{j \neq i} \widetilde{d_{j} G} \circ g\right) \leq(N-1) \sum_{i \neq j} \operatorname{Var}\left(\widetilde{d_{j} G} \circ g\right),
$$

where the last inequality is a well-known inequality for the variance of the sum of random variables. So,

$$
R_{i}(G) \leq(N-1) \sum_{j \neq i} R_{j}(G)
$$

QED

This result suggests that the uncertainty of a game has a component that is independent of the strategic situation of an individual. In large games the results of similar type imply that even if a player does not know his exact role in a game, he is able to evaluate the probable range of the risk that he is going to encounter. Therefore, the player could meaningfully compare games on the basis of their uncertainty, or riskiness. A possible measure of expected uncertainty of a game is the average of the players' uncertainties:

$$
R(G)=\frac{1}{N} \sum_{i=1}^{N} R_{i}(G) .
$$

It is the uncertainty that a player can expect to encounter if he is not yet aware of his role in the game.

The properties above have been mostly concerned with comparison of the Shapley uncertainties for the different players. Another important property, 
convexity, is concerned with behavior of the uncertainty relative to the addition of games.

Proposition 4.6 (Convexity) For each pair of games $G$ and $H$,

$$
R_{i}(\alpha G+(1-\alpha) H) \leq \alpha R_{i}(G)+(1-\alpha) R_{i}(H)
$$

The equality is only possible if the games are proportionate to each other.

Proof: The uncertainty of a game is the variance of a random variable. The assertion of the proposition follows from the well-known property of the variance of a weighted sum of two random variables. QED

From this property the following upper bound on the Shapley uncertainty of the sum of two games follows.

Corollary 4.1 For each pair of games $G$ and $H$,

$$
R_{i}(G+H) \leq 2\left[R_{i}(G)+R_{i}(H)\right]
$$

The equality is only possible if the games are proportionate to each other.

Corollary 4.1 gives an upper bound to the uncertainty of the sum of two games. What can be said about the lower bound? The question boils down to whether two games with large Shapley uncertainty can add up to a game with low uncertainty. Numerical experiments suggest the following conjecture:

Conjecture 4.1 There exist such $d(n)>0$ that for each pair of superadditive n-player games $G$ and $H$

$$
R_{i}(G+H) \geq d(n)\left(R_{i}(G)+R_{i}(H)\right)
$$


This conjecture is true for symmetric convex games. In these games, the worth of a coalition depends only on its size and the dependence is non-decreasing and convex:

$$
G(S)=g(|S|), \text { where } g^{\prime}(x) \geq 0, \text { and } g^{\prime \prime}(x) \geq 0
$$

For these games, the following proposition holds

Proposition 4.7 For each pair of symmetric convex games $G$ and $H$,

$$
R_{i}(G+H) \geq R_{i}(G)+R_{i}(H)
$$

Proof: Clearly the proposition holds if player $i$ 's marginal contributions have positive covariance for each pair of symmetric convex games $G$ and $H$ :

$$
\operatorname{Cov}\left\{d_{i} G(S), d_{i} H(S)\right\} \geq 0
$$

where $S$ is the random coalition. In other words, it is sufficient to prove that

$$
\mathrm{E}\left\{d_{i} G(S) d_{i} H(S)\right\}-\mathrm{E}\left\{d_{i} G(S)\right\} \mathrm{E}\left\{d_{i} H(S)\right\} \geq 0 .
$$

Marginal contributions $d_{i} G(S)$ and $d_{i} H(S)$ are non-negative and non-decreasing in argument $S$, and therefore inequality (4.8) holds because of the following lemma:

Lemma 4.1 If $f_{1}(x)$ and $f_{2}(x)$ are two non-negative, non-decreasing functions, and $\mathcal{F}(x)$ is the cumulative distribution function of a random variable, then

$$
\int_{-\infty}^{\infty} f_{1}(x) f_{2}(x) d \mathcal{F}(x) \geq\left(\int_{-\infty}^{\infty} f_{1}(x) d \mathcal{F}(x)\right)\left(\int_{-\infty}^{\infty} f_{2}(x) d \mathcal{F}(x)\right)
$$


Proof of Lemma: Note that if the lemma holds for $\left\{f_{1}^{\prime}, f_{2}\right\}$ and $\left\{f_{1}^{\prime \prime}, f_{2}\right\}$, then it also holds for $\left\{\alpha^{\prime} f_{1}^{\prime}+\alpha^{\prime \prime} f_{1}^{\prime \prime}, f_{2}\right\}$, where $\alpha^{\prime}$ and $\alpha^{\prime \prime}$ are non-negative coefficients. Similarly, if the lemma holds for $\left\{f_{1}, f_{2}^{\prime}\right\}$ and $\left\{f_{1}, f_{2}^{\prime \prime}\right\}$, then it also holds for $\left\{f_{1}, \beta^{\prime} f_{2}^{\prime}+\beta^{\prime \prime} f_{2}^{\prime \prime}\right\}$, where $\beta^{\prime}$ and $\beta^{\prime \prime}$ are non-negative. It follows that the lemma needs only to be proved for a set of elementary functions that can approximate all non-negative, non-decreasing functions by linear combinations with non-negative coefficients. It is enough to take the set of the following elementary functions $\phi_{T}$ :

$$
\phi_{T}(x)= \begin{cases}1 & \text { if } x \geq T, \\ 0 & \text { if } x<T,\end{cases}
$$

which are characteristic functions of sets $[T ; \infty)$.

Define measure $\mu_{\mathcal{F}}$ as follows:

$$
\mu_{\mathcal{F}}\{X\}=\int_{-\infty}^{\infty} \chi_{X}(x) d \mathcal{F}(x)
$$

where $\chi_{X}(x)$ denotes the characteristic function of set $X$.

In terms of this measure, the inequality for elementary functions becomes:

$$
\mu_{\mathcal{F}}\left\{\left[T_{1}, \infty\right) \cap\left[T_{2}, \infty\right)\right\} \geq \mu_{\mathcal{F}}\left\{\left[T_{1}, \infty\right)\right\} \mu_{\mathcal{F}}\left\{\left[T_{2}, \infty\right)\right\}
$$

which is evidently true because $\left[T_{1}, \infty\right) \cap\left[T_{2}, \infty\right)$ is either $\left[T_{1}, \infty\right)$ or $\left[T_{2}, \infty\right)$, and $\mu_{\mathcal{F}}\{X\} \leq 1$ for any $X$. QED.

\section{Conclusion}

In the course of this paper, the concept of the Shapley uncertainty has been rigorously defined and illustrated by means of several examples. Certain prop- 
erties of this concept have been proved that show how the uncertainty changes under scaling in the size of the game, substitution in the role of players, and taking an average of games. These examples and propositions illustrate that the Shapley uncertainty is an object, that has many interesting properties. The main question is, however: What is this concept good for?

Actually, it is good for several important things. First, it measures the reliability of Shapley value solutions to various practical problems. Second, it is useful in checking the robustness of theoretical arguments that employ the Shapley value as the embodiment of the outcome in market games. Third, the Shapley uncertainty is an additional dimension that a player should take into account if he evaluates the prospect of playing a game. Finally, the Shapley uncertainty may be helpful in the design of games as implementation mechanisms, where the designer is interested in ensuring the stability of the outcome and making the mechanism attractive to risk-sensitive participants.

\section{References}

Aumann, R. J. (1975). Value of markets with a continuum of traders. Econometrica 43(4), 611-646.

Evans, R. A. (1996). Value, consistency, and random coalition formation. Games and Economic Behavior 12, 68-80.

Gul, F. (1989). Bargaining foundations of Shapley value. Econometrica 5\%, $81-95$. 
Hart, S. and A. Mas-Colell (1996). Bargaining and value. Econometrica 64, $357-380$.

Land, M. and O. Gefeller (1997). A game-theoretic approach to partitioning attributable risks in epidemiology. Biometrical Journal 39(7), 777-792.

Osborne, M. J. and A. Rubinstein (1994). A Course in Game Theory (1 ed.). The MIT Press.

Roth, A. E. (1977a). Bargaining ability, the utility of playing a game, and models of coalition formation. Journal of Mathematical Psychology 16, $153-160$.

Roth, A. E. (1977b). The Shapley value as a von Neumann-Morgenstern utility. Econometrica 45(3), 657-664.

Roth, A. E. (1988). The expected utility of playing a game. In A. E. Roth (Ed.), The Shapley Value, pp. 51-70. Cambridge University Press.

Rubinstein, A. (1982). Perfect equilibrium in a bargaining model. Econometrica 50(1), 97-110.

Shapley, L. S. (1953). A value for n-person games. In H. W. Kuhn and A. W. Tucker (Eds.), Contributions to the Theory of Games, Volume 2, pp. 307317. Princeton: Princeton University Press. (Annals of Mathematics Studies, 28).

Shapley, L. S. and M. Shubik (1967). Ownership and the production function. Quarterly Journal of Economics 81(1), 88-111.

Weber, R. J. (1988). Probabilistic values for games. In A. E. Roth (Ed.), The 
Shapley Value, pp. 101-119. Cambridge University Press. 